\title{
Theoretical study of collimated field emission of electrons from a point source
}

\author{
E. Tekman and S. Ciraci \\ Department of Physics, Bilkent University, Bilkent, 06533 Ankara, Turkey
}

\author{
A. Baratoff \\ IBM Research Division, Zurich Research Laboratory, CH-8803 Rüschlikon, Switzerland
}

(Received 26 June 1990; revised manuscript received 29 August 1990)

\begin{abstract}
We clarify basic mechanisms for collimated field emission of electrons from a metallic tip of atomic dimensions. The effective potential barriers arising from the lateral confinement of current carrying states efficiently suppress states with higher transverse quantum numbers. The hornlike opening of the three-dimesional potential improves collimation even if the transmission is not adiabatic. We also find field-dependent resonance and diffraction effects.
\end{abstract}

Recently, Fink' achieved the fabrication of stable W(111) tips terminated by three or even one atom, thus providing a charged-particle sources of atomic dimensions. A stable low-energy electron beam with current $I$ up to $\sim 10 \mu \mathrm{A}$ can be obtained by using much lower voltages (a few $100 \mathrm{~V}$ ) than with conventional field emitters. Moreover, in spite of the finite transverse momentum of the incident electrons, the beam is well collimated, its angular halfwidth being as small as $\sim 2^{\circ}$ at the collector screen. Very recently, interference fringes due to scattering of a similar beam by carbon fibers have demonstrated sufficient coherence to perform holography with lowenergy electrons. ${ }^{1}$

A proper understanding of the effects producing such a collimated and coherent electron beam has been the major issue. The collimation of the electrons in semiconductor microstructures has been treated earlier in the context of point-contact resistance ${ }^{2}$ and of Hall-effect quenching. ${ }^{3}$ In those systems, the dimensionality and, especially the (ballistic) character of the transport were, however, quite different from the present case. Recent theoretical studies $^{4,5}$ on field emission from atomic-size sources arrived at different conclusions for the mechanisms of collimation. In the studies by García and co-workers ${ }^{4}$ the source was simulated by a quasi-one-dimensional constriction connected to a free-electron reservoir, and the effect of the applied field $F$ was represented by a potential barrier of constant height abutting this constriction. They found that the potential barrier is exclusively responsible for the collimation, a plane triangular barrier being particularly efficient. On the other hand, Lang, Yacoby, and Imry ${ }^{5}$ noted that owing to the enhanced field near the tip, a potential channel with a hornlike profile forms in front of its apex. In this waveguide the single-particle wave functions were claimed to evolve adiabatically, ${ }^{6}$ so that their longitudinal momentum increases without reflections and scattering among subbands (modes). The effective barrier in the channel filters incident states by selecting those with small transverse momentum. Collimation was attributed to the barrier and to the adiabatic evolution of the states in the hornlike channel opening. ${ }^{5}$ By themselves, however, the self-consistent-field (SCF) jellium calculations by Lang et al. ${ }^{5}$ did not provide evidence for adiabati- city in situations where relevant dimensions are comparable to $\lambda_{F} \sim 3.5 \AA$. For a single-atom tip this occurs in fields $F \sim 1 \mathrm{~V} / \AA$ required for high currents.

In this Rapid Communication, we present the results of a model study, which resolve those controversial issues and enable a systematic analysis of the influence of different parameters (e.g., $F$, tip work function, channel width, and flaring) or relevant properties of the emitted beam. In particular, we clarify basic mechanisms leading to the collimation and find that the mode (subband) selection by the effective barrier due to transverse confinement of current carrying states is indeed essential for atomicsize emitters. The electric field and the hornlike opening improve the collimation even though the adiabatic is not valid. In experiments, the electrode or screen to which the voltage is applied is placed centimeters to fractions of $\mu \mathrm{m}$ away, but the intrinsic quantum phenomena of interest occur only within a few $\lambda_{F}$ of the tip. Additional collimation due to the curvature of electron trajectories in the region beyond ${ }^{7}$ is not considered here.

The three-dimensional (3D) potential in the vicinity of the tip is represented by

$$
V(F ; \rho, z)=\phi_{m}(F, z)+\alpha(z) \rho^{2} \theta\left(z+l_{t}\right) \theta(d-z),
$$

where $\phi_{m}(F, z)$ is the bimetallic junction potential calculated for two parallel jellium electrodes with edges at $z=0$ (tip) and $z=L$. The electric field is incorporated following Orosz and Balázs. ${ }^{8}$ In the region between the base of the tip $\left(z<-l_{t}\right)$ and the outer vacuum region where lateral variations become negligible $(z>d)$, the potential is assumed parabolic in the transverse direction, thus defining a channel in front of the apex of the tip. As schematically illustrated in Fig. 1, Eq. (1) can also describe the hornlike shape of the potential on the tip side and on the vacuum side. This is achieved by uniformly varying the lateral extend of the confining potential, $w=\left(\hbar^{2} / 2 m \alpha\right)^{1 / 4}$ in the intervals $\left(-l_{t},-l_{1}\right)$ and $\left(l_{2}, d\right)$. Earlier, a similar type of potential for $F=0$ was derived from SCF pseudopotential calculations ${ }^{9}$ and used to analyze the character of transport ${ }^{10}$ as a function of tipsample distance in scanning tunneling microscopy. The form of the potential Eq. (1) is in compliance with the results of SCF calculations, ${ }^{5}$ as may be judged by compar- 

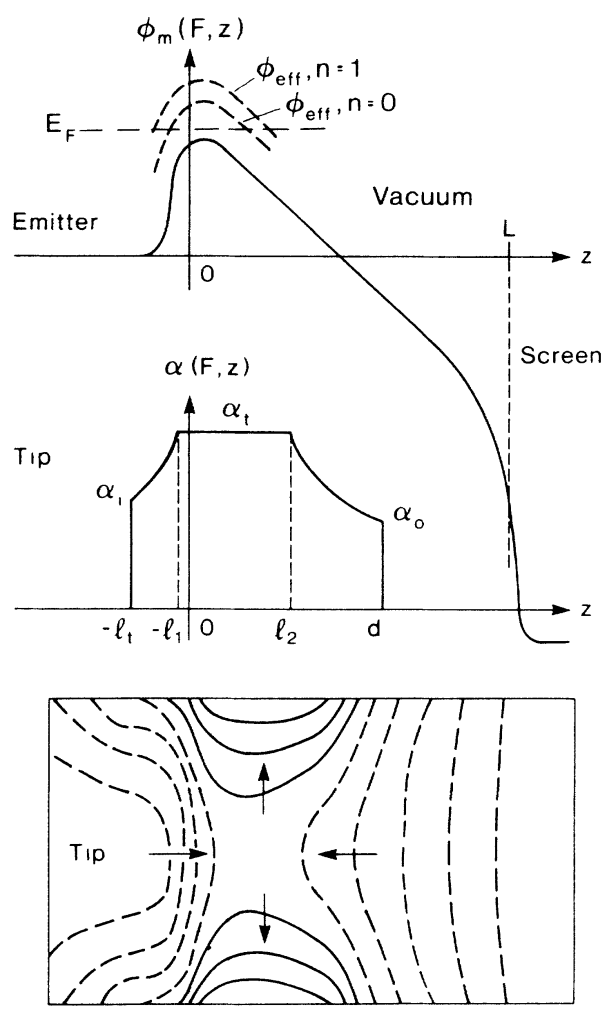

FIG. 1. Top: schematic illustration of $\phi_{m}(F, z), n$-dependent $\phi_{\mathrm{eff}}(F, z), \alpha(z)$, and geometrical parameters. Bottom: contours of constant potential $V(F ; \rho, z)$ calculated in a plane $\rho \leq 4.25 \AA$ and $-4 \leq z \leq 10 \AA$ for $\alpha_{i}=0.2, \alpha_{t}=0.5, \alpha_{0}=0.02 \mathrm{eV} / \AA^{2}$, and $F=2 \mathrm{~V} / \AA$. The contour spacing is $2.5 \mathrm{eV}$; dashed lines are for $V(F ; \rho, z)<E_{F}$. Throughout this work geometrical parameters, $l_{t}=4 \AA, d=10 \AA, L=30 \AA$, and electronic parameters, $E_{F} \sim 12.5 \mathrm{eV}$ and tip work function $\phi \sim 5 \mathrm{eV}$ corresponding to aluminum jellium are used.

ing Fig. 5 of Ref. 5 with the contours of $V(F ; \rho, z)$ shown in Fig. 1 for particular parameter values. In reality $\alpha$ increases and thus the lateral confinement becomes pronounced as the size of the tip apex is decreased or $F$ is increased, but it is convenient to conceptually separate longitudinal effects contained in $\phi_{m}(F, z)$ from transverse ones. In the present model the channel modifies the potential barrier and the transmission in the presence of $F$ is treated quantum mechanically across and behind the barrier up to $z=d$, and semiclassically beyond. By contrast, in the models of García and co-workers ${ }^{4}$ the channel precedes the potential barrier in space, and the motion of the electrons is sometimes treated classically just beyond the turning point. ${ }^{7}$

Using the transfer-matrix method described by Tekman and Ciraci, " we obtain current carrying solutions $\psi_{\mathbf{k}_{\mathbf{k}}}$, corresponding to an incident wave vector $\mathbf{k}_{i}$ deep in the emitter. To this end, we divide the region $-l_{t} \leq z \leq d$ into discrete segments, in which $\phi_{m}(F, z)$ and $\alpha(z)$ can be assumed constant. In each segment the wave functions have the form

$\psi_{\mathbf{k}_{i}}(\rho, z)=\sum_{n}\left[A_{n \mathbf{k}_{i}}(z) e^{i \gamma_{n}(z) z}+B_{n k_{i}}(z) e^{-i \gamma_{n}(z) z}\right] \Phi_{n}(\rho, z)$, where

$$
\gamma_{n}(z)=\left\{\left(2 m / \hbar^{2}\right)\left[E-\phi_{m}(F, z)-\varepsilon_{n}(z)\right]\right\}^{1 / 2}
$$

is the propagation constant for the $n$th $\left(n=n_{x}+n_{y}\right)$ subband state quantized in the channel with energy $\varepsilon_{n}(z)=(n+1) \hbar^{2} / m w^{2}(z)$ and harmonic oscillator eigenfunction $\Phi_{n}(\rho, z) \propto \exp \left[-\rho^{2} / 2 w^{2}(z)\right]$. The coefficients $\boldsymbol{A}_{\boldsymbol{n} \mathbf{k}_{i}}$ and $\boldsymbol{B}_{\boldsymbol{n} \mathbf{k}_{i}}$ are determined by imposing the usual continuity requirements at the boundaries between the segments and at $z=-l_{t}$ and $z=d$. The current energy distribution is derived from the expectation value of the current-density operator,

$J(F, E)=(2 \pi)^{-3} \int d \mathbf{k}_{i}\left\langle\psi_{\mathbf{k}_{i}}\left|\hat{j}_{z}\right| \psi_{\mathbf{k}_{i}}\right\rangle\left(\hbar^{2} k_{i}^{2} / 2 m-E\right)$,

and its angular spread

$\Omega(F, E)=\tan ^{-1}\left(\frac{\left\langle\kappa^{2}(E)\right\rangle}{\left(2 m / \hbar^{2}\right)\left[E-\phi_{m}\left(F, z_{c}\right)\right]-\left\langle\kappa^{2}(E)\right\rangle}\right)^{1 / 2}$

is defined in terms of the expectation value of the transverse wave vector squared $\left\langle\kappa^{2}(E)\right\rangle$ at a point $z_{c}$ slightly to the left of $z=L$. Neglecting thermal broadening, the total emission current is expressed by $I(F)=\int_{0}^{E_{F}} d E J(F, E)$; the energy spread of the emitted beam is specified by $\Delta E=\int_{0}^{E_{F}} d E\left(E_{F}-E\right) J(F, E) / I(F)$, and its collimation angle by

$$
\theta_{c}(F)=\int_{0}^{E_{F}} d E \Omega(F, E) J(F, E) / I(F) .
$$

Note that collimation effects due to effective barrier, the horn, and the electric field are taken into account. On the other hand, the emission angle $\theta_{e}(F)$ similarly defined at $z=d$ excludes the semiclassical collimation effect due to the electric field beyond the horn. ${ }^{\text {? }}$

In order to reveal the effects of transverse confinement we first assume that $\alpha$ in Eq. (1) is constant throughout the range $-l_{t} \leq z \leq d$ (i.e., a uniform channel). In Fig. 2(a) the variation of $I$ with $F$ is illustrated for several values of $\alpha$. For fixed $\alpha, I$ increases with increasing $F$, since the height and thickness of the tunneling barrier decrease. At large $F, \phi_{m}(F, z)$ is eventually depressed below $E_{F}$, as illustrated in Fig. 1, the lowest effective barrier $\phi_{\mathrm{eff}}(F, z) \sim\left[\varepsilon_{0}(z)+\phi_{m}(F, z)-E_{F}\right]$ almost disappears, so that $I(F)$ tends to level off. On the other hand, the effective emission area decreases and subbands (channel modes) shift upward in energy with increasing $\alpha$; consequently the corresponding effective barriers ${ }^{11}$ increase; this causes $I$ to decrease for fixed $F$.

The net effects of $F$ and $\alpha$ on $\theta_{c}$ are summarized in Fig. 2(b): the collimation is improved by decreasing confinement (i.e., increasing the source size) and also by increasing $F$. We attribute these trends to reduced diffraction at the end of the channel and to the more rapid increase of the longitudinal wave vector $\gamma_{n}=0(z)$ beyond the effective barrier. By themselves, the concomitant decrease in barrier height and thickness would give rise to the opposite trends. Moreover, as emphasized in Ref. 4, these trends would be unaffected if the channel preceded the barrier. Such a situaiton may well be realized in semi- 

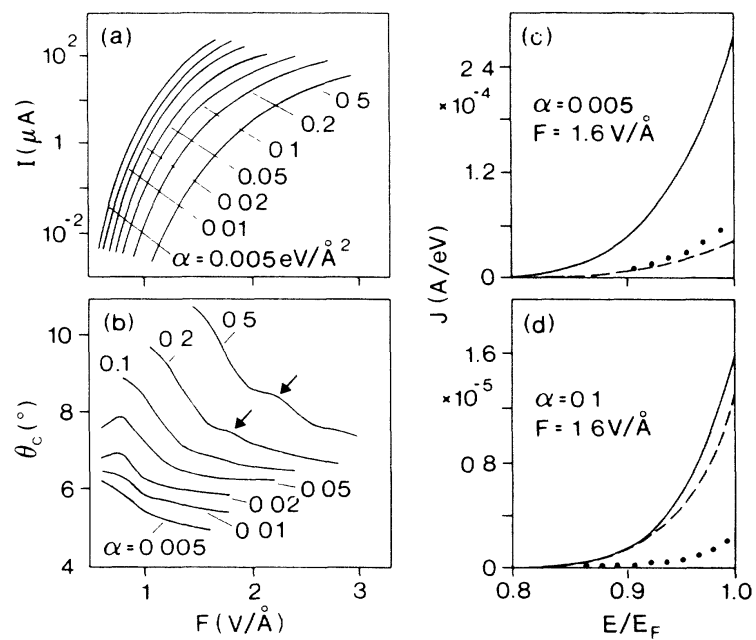

FIG. 2. (a) Emission current $I$ and (b) collimation angle $\theta_{c}$ at $z=z_{c}$ vs electric field $F$ for uniform channels $\left(\alpha_{i}=\alpha_{i}\right.$ $=\alpha_{0}=\alpha$ ). Current energy distribution $J(F, E)$ is shown for (c) $\alpha=0.005 \mathrm{eV} / \AA^{2}$, and (d) $\alpha=0.1 \mathrm{eV} / \AA^{2}$; the contribution of first $n=0$ (second $n=1$ ) mode is shown by the dashed (dotted) line.

conductor microstructure, ${ }^{4}$ but does not apply in our case. It would be highly desirable to test these differing predictions. Care should be taken to measure a quantity similar to $\theta_{c}$, e.g., the angular width at half maximum. The apparent size of the beam spot above a certain detection level may show spurious trends. One must also keep in mind that the above-mentioned increase of $\alpha$ with $F$ might give rise to an increase in $\theta_{c}$, followed by saturation at high $F$. Furthermore, for a somewhat broader facet at the apex of the tip, the field will be enhanced at its edge or corners, so that our model no longer applies.

The weak features in $\theta_{c}(F)$ curves found at high $\alpha$ and $F$ [shown by arrows in Fig. 2(b)], arise from matching to field resonances, i.e., approximately standing-wave solution between the outer edge of the barrier and the partially reflecting channel end. ${ }^{11,12}$ The resulting modulation of the transmitted diffraction pattern gives rise to structure in $\Omega(F, E)$ and $\theta_{c}(F)$. While $J(F, E)$ and $I(F)$ are devoid of any visible structure, $d^{2}\left(\log _{10} J\right) / d E^{2}$ exhibits oscillations shifting with $F$.

In Figs. 2(c) and 2(d) the current energy distribution $J(F, E)$ is shown for widely different values of $\alpha$, together with the contributions from the lowest two subbands. It is clear that the mode selection improves with increasing $\alpha$ due to the increased subband separation, $\left(\varepsilon_{1}-\varepsilon_{0}\right)$. In Fig. 2(c) the $n=1$ contribution exceeds that from the $n=0$ mode because the former is doubly degenerate. Since the energy spread $\Delta E$ is determined by tunneling, it increases with increasing $F$ and decreasing $\alpha$ (i.e., with increasing I). A large current and a small $\Delta E$ (less than $\sim 0.5 \mathrm{eV}$ ) are mutally exclusive. The effect of $\alpha$ due to mode selection within the barrier is essentially absent if the channel precedes the latter. ${ }^{4}$ The effects of mode selection on collimation are more subtle because $\theta_{c}$ appears dominated by the growth of $\gamma_{n}(z)$ and by diffraction beyond the barrier.

We next consider the effects of the hornlike opening into the outer region, which is described by the parame- ters $\alpha_{t}, \alpha_{0}$, and $l_{2}$ in Fig. 1. These effects are examined by comparing the results obtained for two horn structures with those calculated for the corresponding uniform channels with $\alpha=\alpha_{t}$ or $\alpha_{0}$, as shown in Fig. 3. The effective barrier with the horn is higher than that for $\alpha=\alpha_{0}$, but only slightly thinner than that for $\alpha=\alpha_{t}$. Consequently $I$ is significantly higher for $\alpha=\alpha_{0}$, but approaches that obtained for the uniform channel with $\alpha=\alpha_{t}$ for large $F$ as seen in Figs. 3(a) and 3(d). The contribution of the two lowest modes $(n=0$ and $n=1)$ to the total emission current, calculated at $z=d$, is illustrated in Figs. 3(b) and 3(e). Obviously, the suppression of the higher modes is more complete if the channel is narrower either along its whole length or its central portion. The deterioration of mode selection with increasing $F$ arises because the ratio $\left(\gamma_{1}-\gamma_{0}\right) / \gamma_{0}$ then becomes increasingly smaller beyond the higher effective barrier.

Compared to the uniform channel with $\alpha=\alpha_{t}$, the effect of the horn on mode selection is small and more pronounced at low $F$, but the improvement in collimation apparent in Figs. $3(\mathrm{c})$ and $3(\mathrm{f})$ is quite dramatic. An analysis of the relative contributions from different modes in different segments along the horn reveals that their ratio is nearly invariant. At first sight the explanation of collimation by Lang et al. ${ }^{5}$ in terms of mode selection inside the barrier followed by nearly perfect transmission at

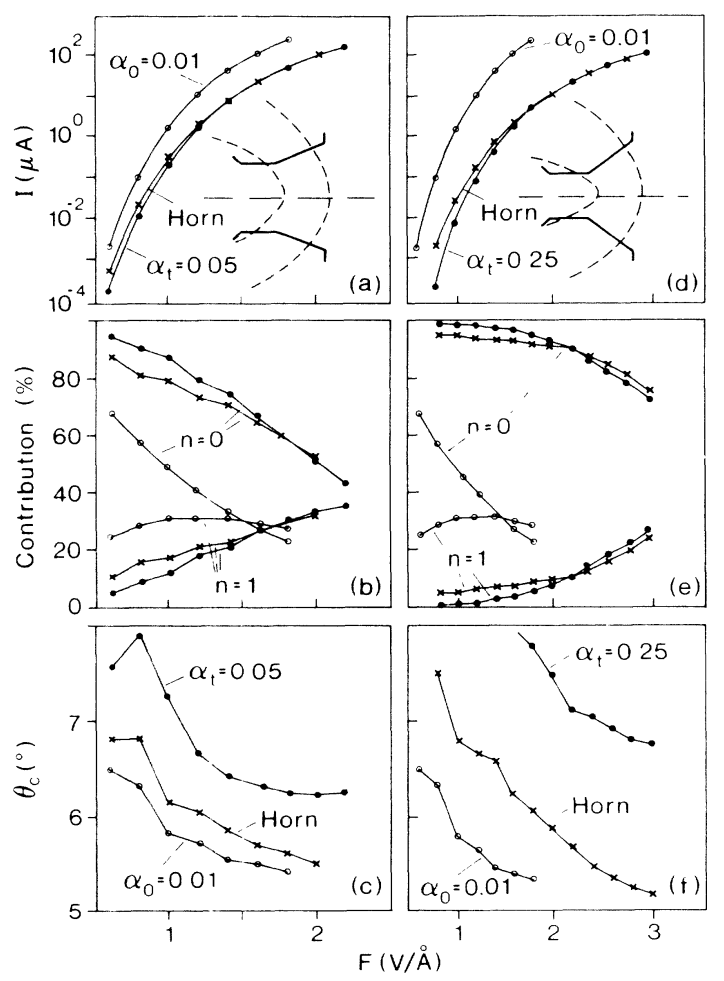

FIG. 3. (a),(d) Emission current $I$, (b),(e) contributions from the $n=0$ and $n=1$ modes evaluated at $z=d$. (c),(f) collimation angle $\theta_{c}$ at $z=z_{c}$ for two horn geometries (see insets) shown by the lines through crosses. The left- (right-) hand-side panel corresponds to $\alpha_{t}=0.05\left(\alpha_{t}=0.25\right) ; \alpha_{0}=0.01 \mathrm{eV} / \AA^{2}, l_{2}=4 \AA$. The open (solid) circles correspond to the same quantities for the uniform channels with $\alpha=\alpha_{0}\left(\alpha_{t}\right)$. 
its end appears correct. However, such an adiabatic evolution takes place if the variation of the channel with $w$ is small on the scale of the electron wavelength. This is not the case, especially for the more flared horn with $\alpha_{t}$ $=0.25$. If the adiabatic picture were valid, the subband states (modes) would evolve slowly and independently. Hence, the total emission current and collimation angle could be obtained by summing only the diagonal matrix elements (with respect to the mode index $n$ ) of the current-density operator $\hat{j}_{z}$ in Eq. (3). For a uniform channel the eigenfunctions $\Phi_{n}(\rho)$ are $z$ independent and thus the diagonal approximation is exact (excluding effects due to reflections). On the other hand, because of the adiabatic reduction of the transverse momentum $\kappa$ along the length of a slowly varying horn, the diagonal approximation is expected to give $\theta_{c}$ smaller than those calculated for the corresponding uniform channels with $\alpha=\alpha_{t}$ and $\alpha=\alpha_{0}$. In our example for the horn structure with $\alpha_{t}=0.25$, the full calculation gives $I=2.04 \mu \mathrm{A}$ and $\theta_{c}=6.2^{\circ}$ for $F=1.6 \mathrm{~V} / \AA$, whereas the diagonal approximation yields $I=1.37 \mu \mathrm{A}$ and $\theta_{c}=3.6^{\circ}$. The values of $\theta_{c}$ calculated for the uniform constrictions with $\alpha=\alpha_{t}$ and $\alpha=\alpha_{0}$ in Figs. $3(\mathrm{c})-3(\mathrm{f})$ are $\sim 8^{\circ}$ and $5.4^{\circ}$, respectively. This demonstrates that in the presence of the horn $\theta_{c}$ is significantly increased owing to intersubband mixing. In view of this analysis and of the results illustrated Figs. $3(c)-3(f)$ we argue that the hornlike potential profile typical for an atomic-size, high-current source improves the collimation, although the adiabatic picture is not valid.

In conclusion, even if the adiabatic approximation does not apply, owing to mode selection near the apex of the tip and confinement in the channel extending beyond the barrier, our model reproduces observed properties of beams emitted from atomic-size tips. We also found fielddependent resonance and diffraction effects.

This work was supported by Joint Project Agreement between Bilkent University and the IBM Zurich Research Laboratory. We thank H. de Raedt, Y. Imry, N. Lang, and J. J. Saenz for supplying copies of their work prior to publication, and H.-W. Fink, R. Morin, and W. Stocker for discussions.
'H. W. Fink, IBM J. Res. Dev. 30, 460 (1986); Phys. Scr. 38, 260 (1988); for the holography see H. W. Fink, W. Stocker, and H. Schmid, Phys. Rev. Lett. 65, 1204 (1990).

${ }^{2}$ C. W. J. Beenakker and H. van Houten, Phys. Rev. B 39, 10445 (1989).

${ }^{3}$ H. U. Baranger and A. D. Stone, Phys. Rev. Lett. 63, 414 (1989).

${ }^{4}$ N. García, J. J. Sáenz, and H. de Raedt, J. Phys. Condens. Matter 1, 9931 (1989); H. de Raedt, N. García, and J. J. Sáenz, Phys. Rev. Lett. 63, 2260 (1989).

${ }^{5}$ N. D. Lang, A. Yacoby, and Y. Imry, Phys. Rev. Lett. 63, 1499 (1989).

${ }^{6}$ L.I. Glazman, G. B. Lesovik, D. E. Khmel'nitskii, and R. I. Shekter, Pisma Zh. Eksp. Teor. Fiz. 48, 218 [Sov. Phys. JETP
48, 238 (1988)]; A. Yacoby and Y. Imry, Phys. Rev. B 41, 5341 (1990).

${ }^{7}$ P. Serena, L. Escapa, J. J. Sáenz, N. Garciá, and H. Rohrer, J. Microsc. (Oxford) 152, 43 (1989).

${ }^{8}$ L. Orosz and E. Balázs, Surf. Sci. 177, 144 (1986).

${ }^{9}$ S. Ciraci, A. Baratoff, and I. P. Batra, Phys. Rev. B 42, 7618 (1990).

${ }^{10}$ S. Ciraci and E. Tekman, Phys. Rev. B 40, 11969 (1989); E. Tekman and S. Ciraci, Phys. Rev. B 42, 1860 (1990).

${ }^{1}$ E. Tekman and S. Ciraci, Phys. Rev. B 39, 8772 (1989); 40, 8559 (1989).

${ }^{12}$ G. Kirczenow, Solid State Commun. 68, 715 (1988); A. Szafer and A. D. Stone, Phys. Rev. Lett. 62, 300 (1989). 